\title{
Difficult subarachnoid anaesthesia Prediction and Performance
}

\author{
André Van Zundert ${ }^{1,2}$ \\ ${ }^{1}$ Department of Anaesthesia, The University of Queensland, Faculty of Medicine, Brisbane, Queensland, Australia. \\ ${ }^{2}$ Department of Anaesthesia and Perioperative Medicine, Royal Brisbane and Women's Hospital, Brisbane, Queensland, Australia.
}

Spinal anaesthesia (SA) has enjoyed a long history of success, celebrating soon its $125^{\text {th }}$ anniversary. Puncturing the dura mater is considered a simple procedure, followed by a subarachnoid injection of a local anaesthetic (LA) agent into the cerebrospinal fluid (CSF). Even when the technique is performed perfectly, there is no guarantee that the block sits perfectly. Failure is not uncommon and encompasses a range from total absence of any neuraxial blockade, a partial block (insufficient height, quality or duration) or a patchy block. Table 1 lists a large number of potential causative factors that may result in a failed spinal anaesthetic, providing suggestions of solutions. Analysing each distinct phase of the procedure, i.e., spinal puncture, injection of local anaesthetic solution, spread of the local anaesthetic solution through the cerebrospinal fluid, action of the drug on subarachnoid neural tissue and patient management, are the keys to success at each stage. Mechanisms of failure of spinal anaesthesia include insufficient preparation and check of equipment and drugs, suboptimal positioning of the patient, unsuccessful puncture due to inadequate training or experience and inadequate use of needles and local anaesthetic solution.[1-5] Besides operator, preparation, technique-dependent and patient-related factors (anatomical variations), there are also organisational factors (lack of block room, lack of adequate monitoring and trained personnel, insufficient time between block and onset of surgery, subsequent management following block). The use of the correct local anaesthetic (dose, volume, concentration) injected at the correct lumbar interspace is of paramount importance to produce an adequate spinal block for the right surgical intervention.

Nevertheless, failures may still occur. Therefore, the anaesthetist should always have a contingency plan for a failed spinal block. Indeed, patients expect reliable surgical anaesthesia when undergoing an operation under regional anaesthesia. If the block fails, we need to be ready to offer a solution, using rescue techniques. The alternatives are either to repeat the spinal anaesthesia or to convert to a general anaesthetic.

Failed spinal anaesthesia has roughly three reasons: a) the local anaesthetic solution does not reach the subarachnoid space; b) the drug has been injected at the right location, but the block is not what is expected; and c) the local anaesthetic solution works well, but the dosage chosen is not correct or results in unilateral or patchy blocks as the resulting block is deficient in quantity, quality or duration.

\section{Ten tips to prevent failed spinal blocks include:}

1. Gentle aspiration of $0.5 \mathrm{ml} \mathrm{CSF}$ before and after injection of local anaesthetic to assure needle point is in the CSF and stayed in the CSF throughout the injection process. This requires a steady hand, stabilising the needle against the back of the patient.

2. Factors influencing subarachnoid spread of the local anaesthetic solutions include: posture of patient immediately following injection, characteristics of local anaesthetic solution (baricity, density and specific gravity of solution, volume, dose, concentration of drug, speed of injection, use of adjuncts such as vasoconstrictors, opioids, clonidine, glucose), patient characteristics (age, weight, height, BMI, volume of CSF, intra-abdominal pressure), variations in technique such as site of injection (insert needle with stylet inside), injection technique (slow, unfractionated vs fractionated or incremental doses), use of introducer, needle size, use of induced turbulence (barbotage).

3. Adequately inform patient about potential side effects, complications and failure of block, providing alternative solutions when obtaining informed consent.

Address of Correspondence: Professor Dr. André Van Zundert, Professor \& Chair Anaesthesiology, The University of Queensland, Faculty of Medicine,

Brisbane, Queensland. Department of Anaesthesia and Perioperative Medicine, Royal Brisbane and Women's Hospital, Brisbane, Queensland, Australia. E-mail: vanzundertandre@gmail.com

DOI: 10.13107/ijra.2021.v02i02.034 | www.ijrajournal.com |

This is an Open Access article distributed under the terms of the Creative Commons Attribution Non-Commercial-Share Alike 4.0 License

(http://creativecommons.org/licenses/by-nc-sa/4.0) which allows others to remix, tweak, and build upon the work non-commercially as long as appropriate credit is given and the new creation are licensed under the identical terms. 


\begin{tabular}{|c|c|c|}
\hline Causative Factor & Difficulties to expect when & Solutions \\
\hline \multirow{4}{*}{ Operator-dependent } & - Lack of adequate training / inexperience & - Adequate results require $>50-70$ blocks \\
\hline & - Lack of continuous experience & - Limit number of attempts \\
\hline & \multirow{2}{*}{ - Lack of smooth insertion technique } & - Ask help from more experienced / supervisor \\
\hline & & - Avoid bone contact \\
\hline \multirow{5}{*}{ Preparation-dependent } & - Lack of block room facility + monitoring & - Allow adequate preparation time \\
\hline & - Lack of adequately trained personnel & - Adequately trained personnel is essential \\
\hline & - Lack of check of equipment and drugs & - Use special needles for spinal anaesthesia \\
\hline & - Lack of adequate equipment / needles & - Quincke vs pencil point needles \\
\hline & $\begin{array}{l}\text { - Lack of adequate sterile precautions } \\
\text { tray }\end{array}$ & $\begin{array}{l}\text { - Anaesthetist should draw up all local anaesthetics, } \\
\text { checked by another member of the team }\end{array}$ \\
\hline \multirow{14}{*}{ Technique-dependent } & - Frequent connections-disconnections & $\begin{array}{l}\text { - Stabilisation of needle during injection against } \\
\text { patient's back }\end{array}$ \\
\hline & - Lack of control of movement of needle & $\begin{array}{l}\text { Especially pencil point needles may see some of } \\
\text { the drug lost into the epidural space }\end{array}$ \\
\hline & $\begin{array}{l}\text { - Incorrect placement of needle tip may cause flap } \\
\text { valve mechanism }\end{array}$ & $\begin{array}{l}\text { - Firm anchoring needle hub between thumb and } \\
\text { forefinger }\end{array}$ \\
\hline & - Subdural injection & $\begin{array}{l}\text { - Subtle misplacements are difficult to } \\
\text { identify/eliminate }\end{array}$ \\
\hline & $\begin{array}{l}\text { - Lack of leak-tight connection between needle } \\
\text { and syringe causing leaks of drugs }\end{array}$ & $\begin{array}{l}\text { - Always evaluate anatomical landmarks (may be } \\
\text { difficult in obese / pregnant patients) }\end{array}$ \\
\hline & - Rotation of needle a full $360^{\circ}$ & - Use smaller syringe for skin infiltration \\
\hline & - Inadequate positioning during/after block & $\begin{array}{l}\text { - Use always the same size for subarachnoid } \\
\text { injections }\end{array}$ \\
\hline & $\begin{array}{l}\text { - Too high insertion risks inadvertent damage to } \\
\text { spinal cord }\end{array}$ & - Use spinal needles of sizes 22-27 gauge \\
\hline & - Use of identical syringe sizes on tray & $\begin{array}{l}\text { - A spinal needle should not be inserted without } \\
\text { the stylet }\end{array}$ \\
\hline & - Inconsistency in syringe sizes on tray & - Blood may also block needle \\
\hline & - Use of a too flexible small needle (e.g., 30 gauge) & \\
\hline & - tends to deviate from midline & \\
\hline & - Use of spinal needle without the stylet & \\
\hline & - Bloody spinal tap & \\
\hline \multirow{6}{*}{ Patient-dependent } & - Extremes of age (very young/very old) & - Adequate patient selection \\
\hline & - Obesity (more difficult if extreme obesity) & - Dose adjustments \\
\hline & - Extreme high/low CSF volume & - Have helper focusing on patient \\
\hline & - Nervousness / psychological diseases & - Have helper for anaesthetist \\
\hline & - Frequent patient movements & - Adequately explain technique to patient - if \\
\hline & - Unwilling / uncooperative patients & needed provide anxiolytic medication \\
\hline \multirow{10}{*}{ Anatomical factors } & - Abnormalities of the spine & - Use information from previous block attempt \\
\hline & - Kyphosis-scoliosis-extreme lordosis & - Use ultrasound-guided technique \\
\hline & - Ankylosis spondylitis & $\begin{array}{l}\text { - Admit sometimes a spinal anaesthesia is not a } \\
\text { good idea for a particular patient with a particular } \\
\text { anatomical deviation }\end{array}$ \\
\hline & - Spinal stenosis & $\begin{array}{l}\text { - Inform patient of potential issues before you start } \\
\text { the block }\end{array}$ \\
\hline & - Previous spinal surgery / chemotherapy & $\begin{array}{l}\text { - Negative correlation CSF volume and peak sensory } \\
\text { height block }\end{array}$ \\
\hline & - Cysts (Tarlov-arachnoid- dermoid) & \multirow{5}{*}{ - Low CSF volume may result in extensive block } \\
\hline & - Marfan's syndrome (dural sac ectasia) & \\
\hline & - Degenerative Disk Disease & \\
\hline & - Compression fractures (osteoporosis) & \\
\hline & - Volume of CSF (extreme low/large) & \\
\hline
\end{tabular}

Table 1 


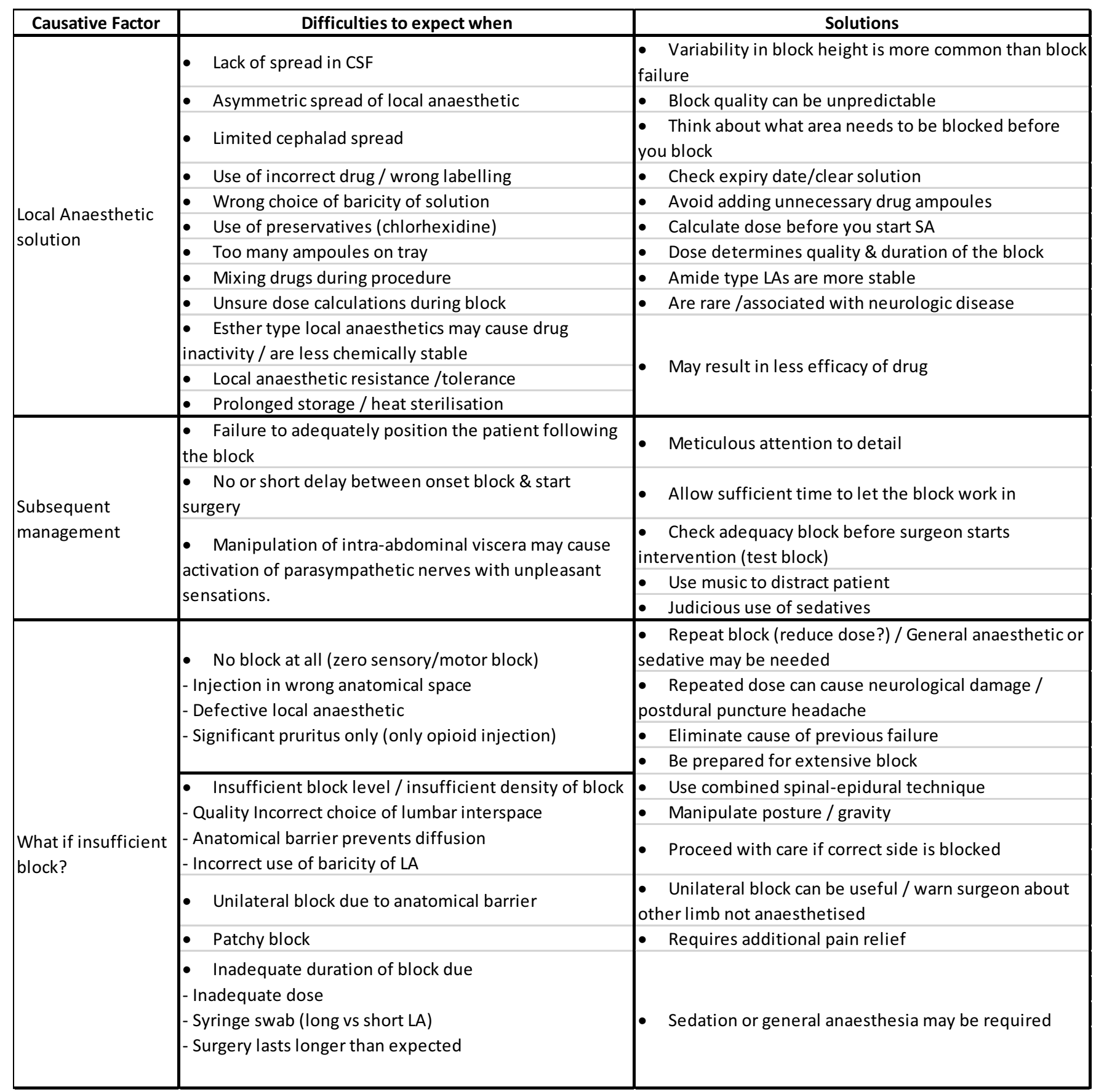

Table 1

4. The time at which failure is detected and the nature of the failure determine the strategy for managing an inadequate spinal anaesthetic. If 10 to 15 minutes have elapsed since the subarachnoid injection and no typical onset pattern of sensory and motor blockade are seen, it is highly unlikely that the spinal anaesthetic will be inadequate for surgery and additional interventions will be required.
Tips to solve the above problems of failed spinal anaesthesia:

1. Be prepared by being honest to yourself, the patient and the surgeon; accept failure; be ready to convert to general anaesthesia. Communication is essential; explain what are the issues; discuss the various options to treat the spinal failure; keep patient and surgeon in the loop. Document everything in detail, including testing results 
(sensory/motor blockade) and attempts to revive the block, increase the extent of the block; the timing of the different sequences; the responses of patient and surgeon. Be prepared to do a postoperative follow-up and provide the patient an opportunity to answer their queries.

2. Avoid excessive and multimodal sedation as it often results in a catastrophe, which you need to avoid.

3. Your approach will be different: a) in case of anaesthesia for a caesarean section (or a vaginal delivery); you need to think about how much more time you have to repeat a block (spinal) or use a combined spinal-anaesthesia technique or insert an epidural catheter. Circumstances may need you to progress fast, without bringing the patient and her baby in danger. Often, a general anaesthetic may be the best solution; b) in case of anaesthesia for a surgical intervention; you need to discuss your options with the surgeon and whether you still can try to repeat the block, or use alternative regional anaesthesia techniques; if not, general anaesthesia may be a good solution.

4. If there is absolute no sign of sensory or motor blockade after 5 or 10 minutes, the cause may be that the LA injection was not in the subarachnoid space/CSF; no LA was injected, but e.g. physiologic saline; or some faulty LA was injected (wrong dose, concentration, volume). Repeat the injection (after a minimal wait of $10 \mathrm{~min}$ ) with caution using minute doses of LA or provide general anaesthesia. If there is an anatomical problem, use the spinal space above or below.

5. If the resulting block is insufficient in height or density due to (dose was not enough; injection site was not correct or too low; patient shows anatomical variations), postural manoeuvres of the operating table can be helpful (pending baricity of the used LA); a supplemental dose of iv opioids or sedatives can make a huge difference - but do not exaggerate in the dose.

6. It there is a unilateral block, due to the wrong position during injection of the local anaesthetic not taking into account the baricity of the LA; or there is an anatomical abnormality; you can use postural manoeuvres (see under point 5) or proceed with care if the correct side is blocked. In case the opposite site is blocked, check your LA (and its baricity). You can use a repeat block (with a lower dose) and make sure that the position this time is correct; otherwise, administer general anaesthesia.

7. In case of patchy blocks (due to a too low dose of LA or anatomical abnormality), repeat the spinal injection, but use a lower dose; add iv opiates/sedatives (moderate doses) or provide general anaesthesia. Be prepared for hypotension after a second/repeat spinal anaesthetic.

8. Inadequate duration (unexpected long duration of an operation) due to insufficient dose of the drug or due to the use of the wrong category of LA, or use of a syringe swap (e.g. using the LA for skin infiltration injected into the subarachnoid space; and the LA intended for spinal anaesthesia injected into the skin); you only can provide extra LA if the patient has a catheter in the neuraxial space; you can discuss whether the surgeon can add some LA in the vicinity of the operative wound; or use moderate doses of opiates/sedatives; or choice to provide general anaesthesia.

9. If the anaesthetist frequently experiences failures of spinal anaesthesia, it is advised to get additional training, including the use of ultrasound-guided anatomical evaluation of the neuraxis.

10. In all cases, safety is the most important rule to follow. If you are uncertain about how best to find a solution for the spinal failure, discuss it with a colleague or use that technique you are most familiar with. Sometimes, this may be a general anaesthetic. One should not be ashamed to admit to that.

If the anaesthetist is well trained, uses a proper technique with meticulous attention to detail, spinal anaesthesia should result in a simple and reliable block with a failure rate that should be less than $1 \%$. Even the best anaesthetist can be faced with a failure as there are many causative factors, which not all can be eliminated during the institution of the block. Therefore, careful assessment of sensory and motor blockade resulting from the spinal blockade needs to be evaluated by the anaesthetist before the surgeon can start the operation. Anaesthetists need to be aware of management strategies in case no, patchy, unilateral or insufficient levels of blocks are the result, which are indicators of a failed spinal anaesthetic. Follow-up procedures must include full documentation of what happened, how the issue was solved, the provision of an explanation to the patient and detailed investigation. 


\section{Question \& Answer with an Expert}

\section{Case 1}

An 80-year-old male was posted for proximal femoral nail of right intertrochanteric fracture. Wt-67 kg / Ht-165 cm / independent / mobile / no signs of cardiac failure / calcified aortic stenosis without pressure gradient / Lab investigations-normal limits. ECG-LBBB and echocardiograph Echo- 42\% ejection fraction with anterolateral wall hypokinesia. Oral LMWH stopped for 5 days and shifted to $\mathrm{s} / \mathrm{c}$ clexane (last dose received 10 hours prior to shifting patient to OT recovery room. As per protocol all patients at time of hip X-rays are also screened for spine.

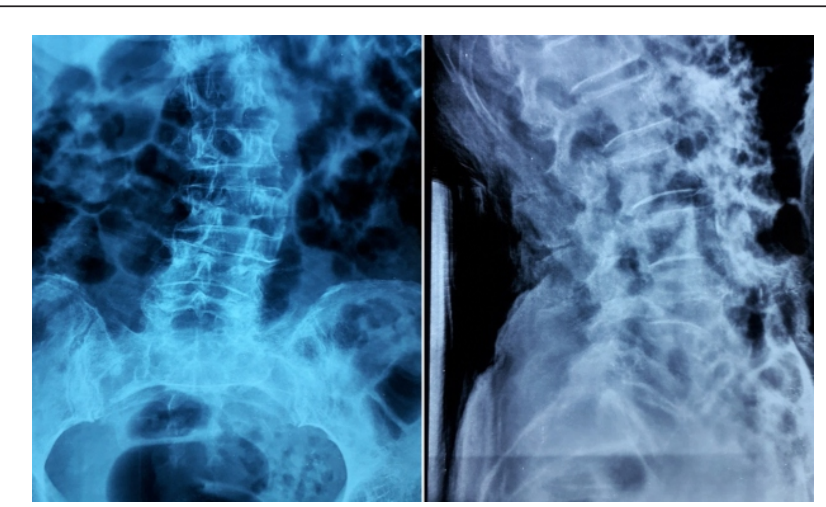

Figure 1: AP and lateral lumbar X-rays.

\section{Questions: Sandeep Diwan}

What would be the anticipated difficulty in spinal anaesthesia and how to overcome these? What space would you choose for needle insertion? Type and gauge? dose of LA? Type and volume of LA? Position of patient for spinal? Medial or paramedian? What if there is no CSF egress? When you think of quitting off? What would be plan B if there a failure?

\section{Answers: Prof Andre Van Zundert}

25 or $27 \mathrm{G}$ pencil point needle

Bupivacaine $0.5 \%$ plain 1.5 to $1.8 \mathrm{ml}$ (depending physical status patient); add small doses of fentanyl or sufentanil or even morphine ( $3^{\text {rd }}$ choice).

Sitting position

L3-L4 SpinalMedian approach - paramedian if not obese If difficult, use CSE technique.

Plan $\mathrm{B}=\mathrm{CSE}$ with epidural catheter and give top up as required to get to an upper sensory block level of T8-T10.

$\mathrm{X}$-rays show scoliosis, but with good interspaces in the lumbar region. I expect to have no difficulties in inserting the spinal needle.
If there is torsio-scoliosis, you need to work out how the torsion is, to make sure that the spinal needle will enter the subarachnoid space.

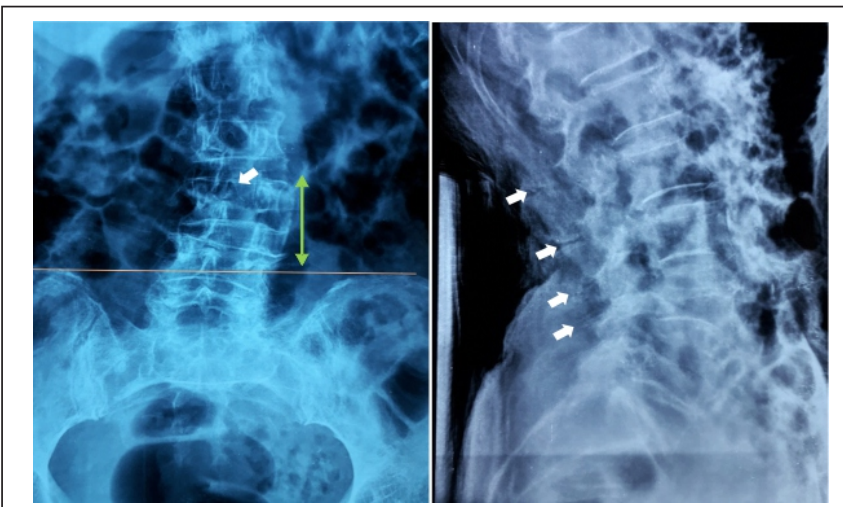

Figure 1: AP and lateral lumbar X-rays.

\section{Case Management: Sandeep Diwan}

The narrow interspinous processes in lateral view (white arrows - LATERAL) would not allow spinal needles, hence a higher paraspinal needle was introduced (white arrow -AP).

Positioning was a problem since the patient was uncooperative. However, a generous local anesthetic infiltration each time you use a different infiltration site is required.

\section{Case 2}

An 84-year-old female, with long standing diabetes mellites and hypertension (<20 years). Wt-76 kg / Ht- $170 \mathrm{~cm} /$ 190/86 mmHg / DM 2/ on insulin actrapid 6/0/6; HbAlc 6 ECG and echocardiography were within normal limits. Xray lumbar spine $\mathrm{AP}$ and lateral were executed prior to the surgery. Lab investigations were normal and she was not on anticoagulants. She suffered from a right subtrochanteric fracture and was to undergo a proximal femoral nail.

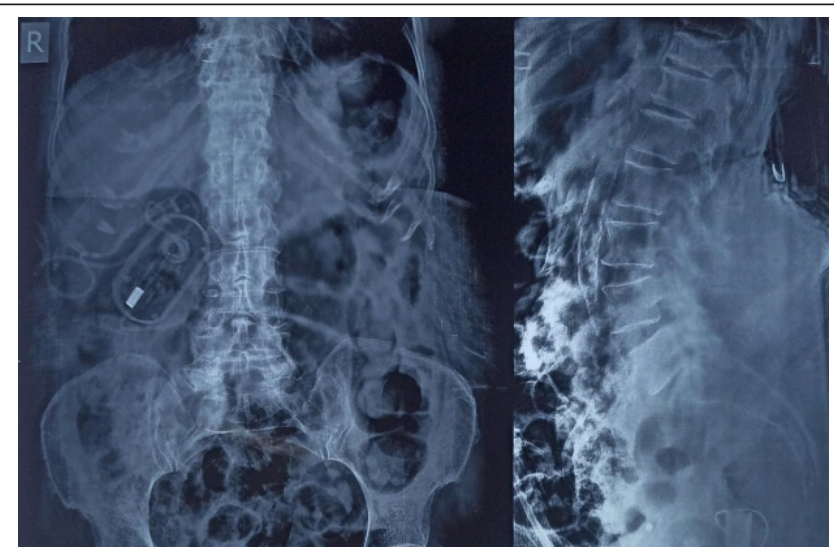

Figure 2: X-ray AP and lateral 


\section{Questions: Sandeep Diwan}

What would be the anticipated difficulty in spinal anaesthesia? and how to overcome these? What space would you choose for needle insertion? Type and gauge? dose of LA? Type and volume of LA? Position of patient for spinal? Medial or paramedian? What if there is no CSF egress? When you think of quitting off? What would be plan B if there a failure?

\section{Answers: Prof Andre Van Zundert}

In this elderly lady, carefully institute spinal block (plain bupivacaine $0.5 \% 1.5 \mathrm{ml}$ plus fentanyl or sufentanil (perhaps morphine if the other 2 are not available). The L34 interspinous process is fine with a $25 \mathrm{~g}$ spinal needle in the sitting position. However, the egress of CSF can be slower. Plan B: CSE.

Plan C: GA ifRA technique fails

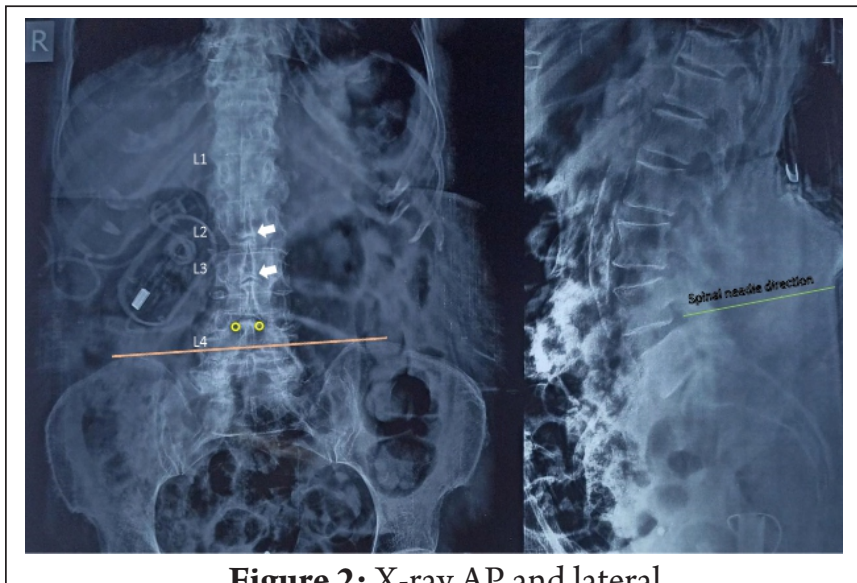

Figure 2: X-ray AP and lateral

\section{Case Management}

Spinal needle could not be inserted at the interspinous levels of L2-3 and L3-4. This could be due to ossification of intervening spinal ligaments. Hence, a L4-5 was chosen and spinal needle direction is noted in lateral view.

\section{Case 3}

\section{Case A}

An 76 year-old-male with intertrochanter fracture for left PFN. Wt- 69 kg / Ht- 163 cm / ABP- 158/56 / ASA 3 / controlled DM and HTN/ Echo- EF= 40\% / not in CCF / on ani-diabetics and hypertension non-dependant and mobile.

\section{Case B}

An 78 year-old-male with intertrochanter fracture for right PFN. Wt- 74kg / Ht- 171cm / ABP- 9\% / not in CCF / on ani-diabetics and hypertension both controlled $\mathrm{h} / \mathrm{o}$ COVID asymptomatic no $\mathrm{O}_{2}$, steroids; non-dependant and mobile. Both had had undergone thoracolumbar and lumbar decompression and instrumentation respectively for a non-malignant spinal cord compression 5-6 years ago. Medical history was $\mathrm{X}$ ray demonstrated a fixation from $\mathrm{L} 2$ S1 in case $3 \mathrm{~A}$ and T9 to L5 in case $3 \mathrm{~B}$.

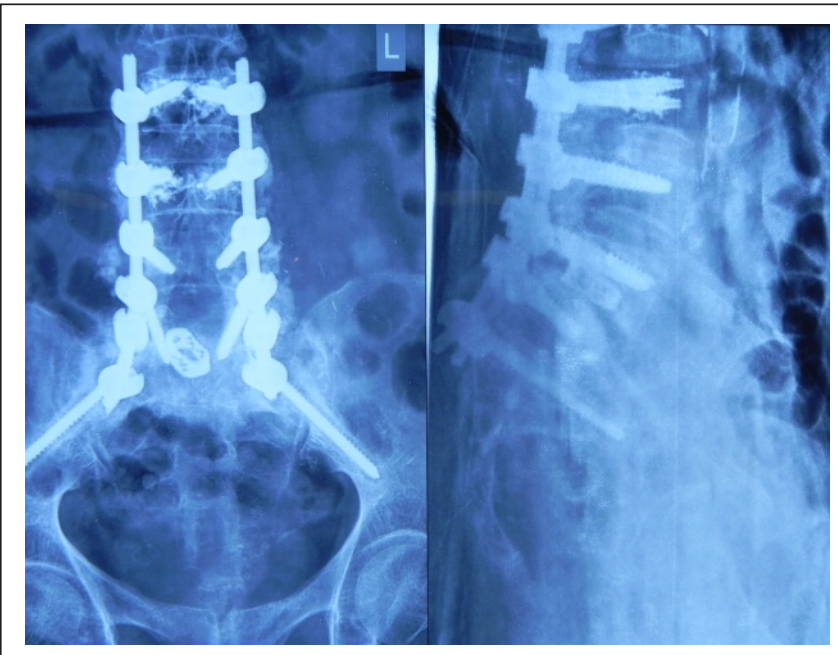

Figure 3A: AP view Figure 3A: Lateral view

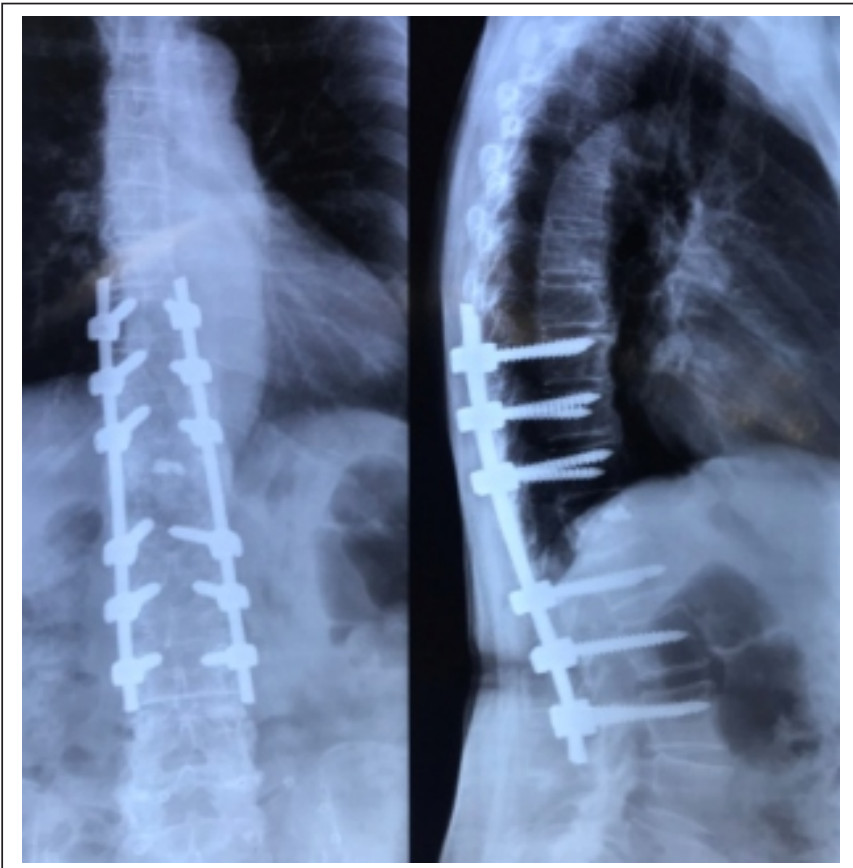

Figure 3B: AP view

Figure 3B: Lateral view

\section{Questions: Sandeep Diwan}

Would you opt for a spinal anesthesia? Do you feel to have an opinion of spine surgeon? Is a long-standing DM a contraindication for a spinal anesthetic? If at all a spinal be administered are their further challenges? What would be the chosen space for spinal? What alternative do we have? 


\section{Answer: Prof Andre Van Zundert}

Spinal anaesthesia is only possible if during the previous operation the region (where we need to puncture) has not been taken in with orthopaedic material. CSE or epidurals not an option as epidural space will be opened during the previous operation. DM is not a contraindication, but bear in mind that the blood pressure and glucose levels may vary substantially during the operation and a careful monitoring is essential. Lumbar and Sacal Plexus block are a viable option.

\section{Case Management}

In our institute, an opinion of spine surgeon is mandatory to exclude possibility of postoperative spinal instrumentation related problems (back pain/radiculopathies/infection etc). Apart from the implants the instillation of bone grafts will hinder spinal needle movement. Positioning for neuraxial anaesthesia was a problem (forward bending). In both patient's lumbosacral plexus block was implemented.

\section{Case 4}

An 90-year-old female of domestic fall suffered from right intertrochanteric fracture. Wt-54 kg / Ht- $139 \mathrm{~cm} / \mathrm{ABP}$ 118/56 / ASA 3 / controlled DM and HTN / Echo- EF= $45 \%$ / not In CCF/ on ani-diabetics and hypertension; dependant but mobile. Apart from pulmonary tuberculosis and frequent cough, rest was normal. On air oxygen saturation was 94\%. Lab investigations and ECG- normal limits. X-ray lumbar spine revealed the following image.

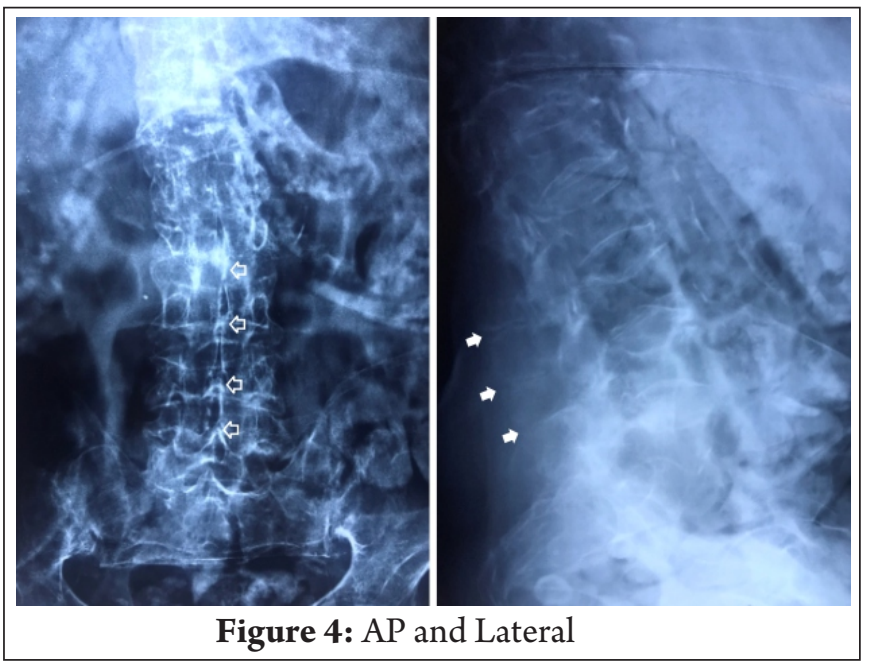

\section{Questions: Sandeep Diwan}

Is spinal a possibility? Is lateral X-ray ok for needle insertion? Do you feel ultrasound has a role in difficult spinal?

\section{Answer: Prof Andre Van Zundert}

All depends on his physical activity at the moment? I certainly would advocate using a spinal. I rather rely on what I feel and not look into the details of xray! I would try spinal with $0.5 \%$ bupi plus fentanyl 20 ug or sufenta 10 ug. I would like to know how long will the operation last? You can also use a combination: N Sciatic Block with a Lumbar Block. US-guided blocks (spinal) in these octogenarians is not easy - who can get enough experience.

Perhaps CSE technique, use catheter top-ups via epidural space to get a sufficient block; catheter can be used in the postop phase.

'These are just impressions, as nothing replaces the physical contact with the patient; and the clinical skills of the anaesthetist who takes care of the patient'. -Andre Van Zundert

\section{Case Management}

The narrow and calcified interspinous processes was difficult for needle insertion. After several attempts a paraspinal needle insertion was possible to tap CSF and accomplish spinal anaesthesia. 


\section{Conflict of interest: Nil Source of support: None}

\section{References}

1. Hadzic A. Mechanisms and management of failed spinal anesthesia. NYSORA. https://www.nysora.com/foundations-of-regionalanesthesia/complications/mechanisms-management-failed-spinalanesthesia/ (accessed 10.06.2021).

2. Alabia AA, Adeniyib OV, Adelekea OA, Pillayc P, Haffajee MR. Factors associated with failed spinal anaesthesia for Caesarean sections in Mthatha general hospital, Eastern Cape, South Africa. S Afr Family Pract 2017;59: 128-132.

3. Fettes PDW, Jansson J-R, Wildsmith JAW. Failed spinal anaesthesia: mechanisms, management, and prevention, Br J Anaesth 2009;102:739-748.

4. Aasvang EK, Laursen MB, Madsen J, Krøigaard M, Solgaard S, Kjaersgaard-Andersen $\mathrm{P}$, et al. Incidence and related factors for intraoperative failed spinal anaesthesia for lower limb arthroplasty. Acta Anaesthesiol Scand. 2018;62:993-1000.

5. Parikh KS, Seetharamaiah S. Approach to failed spinal anaeesthesia for caesarean section. Indian J Anaesth 2018;62:691-7.

How to cite this article: Van Zundert A | Difficult Subarachnoid Anaesthesia Prediction and Performance | International Journal of Regional Anaesthesia | July-December 2021; 2(2): 92-99. 